Revue des patrimoines

4 | 2004

Les réseaux de la villégiature

\title{
Les réseaux de la villégiature : une invitation au voyage
}

\section{Michel Melot}

\section{(2) OpenEdition}

Journals

Édition électronique

URL : http://journals.openedition.org/insitu/2277

DOI : 10.4000/insitu.2277

ISSN : 1630-7305

Éditeur

Ministère de la Culture

Référence électronique

Michel Melot, «Les réseaux de la villégiature : une invitation au voyage », In Situ [En ligne], 4| 2004, mis en ligne le 19 avril 2012, consulté le 07 septembre 2020. URL : http://journals.openedition.org/insitu/ 2277 ; DOI : https://doi.org/10.4000/insitu.2277

Ce document a été généré automatiquement le 7 septembre 2020

\section{(c) (i) (9)}

In Situ Revues des patrimoines est mis à disposition selon les termes de la licence Creative Commons Attribution - Pas d'Utilisation Commerciale - Pas de Modification 4.0 International. 


\title{
Les réseaux de la villégiature : une invitation au voyage
}

\author{
Michel Melot
}

1 Le programme de recherche sur la villégiature n'en finit pas d'ouvrir des perspectives nouvelles sur l'histoire de l'architecture et de l'urbanisme. Les précédentes rencontres avaient mis en évidence le caractère exemplaire, souvent exceptionnel, de cette architecture et de son urbanisme. La villégiature est le lieu de toutes les audaces, parfois de tous les caprices mais toujours l'expression de la demeure personnalisée ou de la cité idéale enfin réalisée.

2 Le thème des réseaux n'est pas moins riche et apporte une nouvelle preuve de la pertinence de ces études dans le cadre d'un programme de recherche du Laboratoire sur le patrimoine français que dirige Claude Mignot. Le mot « réseau » peut prendre plusieurs sens qui tous ont été exposés lors de ces journées de stage où les chercheurs de différentes régions ont pu confronter leurs analyses, comparer leurs méthodes et échafauder de nouvelles hypothèses. 
Figure 1

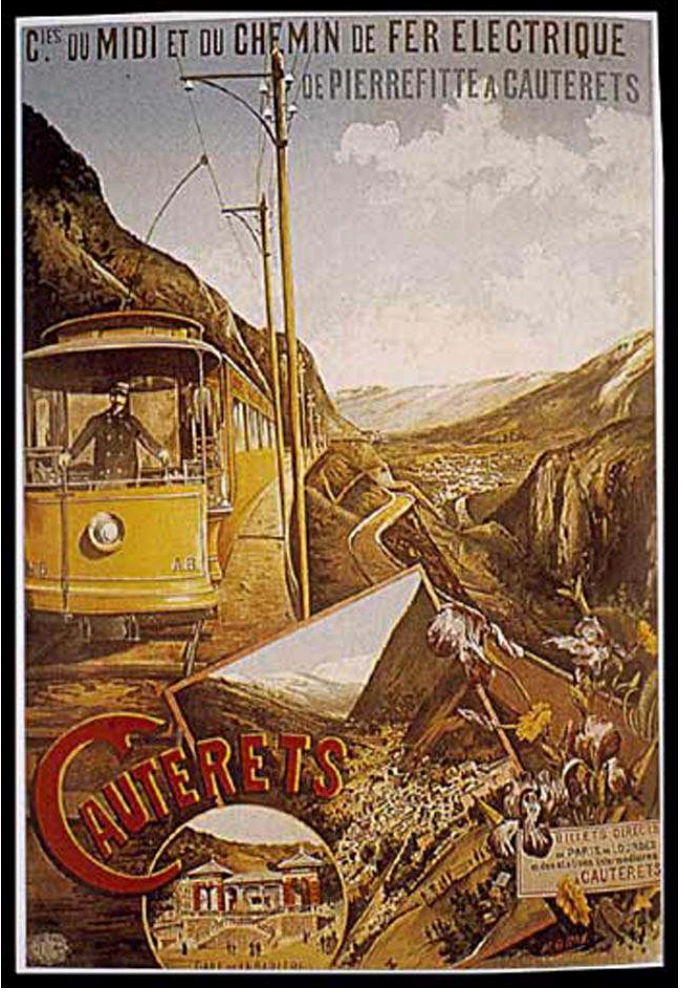

Chemin de fer électrique de Pierrefitte à Cauterets. Compagnie de chemin de fer du Midi. Affiche, s.d. [fin XIXes.]

Coll. part. (c) Droits réservés

3 Au sens le plus trivial, la villégiature est d'abord un formidable pourvoyeur de réseaux de transports (fig. $\mathbf{n}^{\circ} \mathbf{1}$ ). D'une part parce qu'il s'agit de viabiliser des terres parfois vierges du littoral ou des montagnes, mais aussi parce qu'il faut les relier aux villes puisque la villégiature est par définition saisonnière et l'occasion de flux migratoires aujourd'hui considérables. 
Figure 2

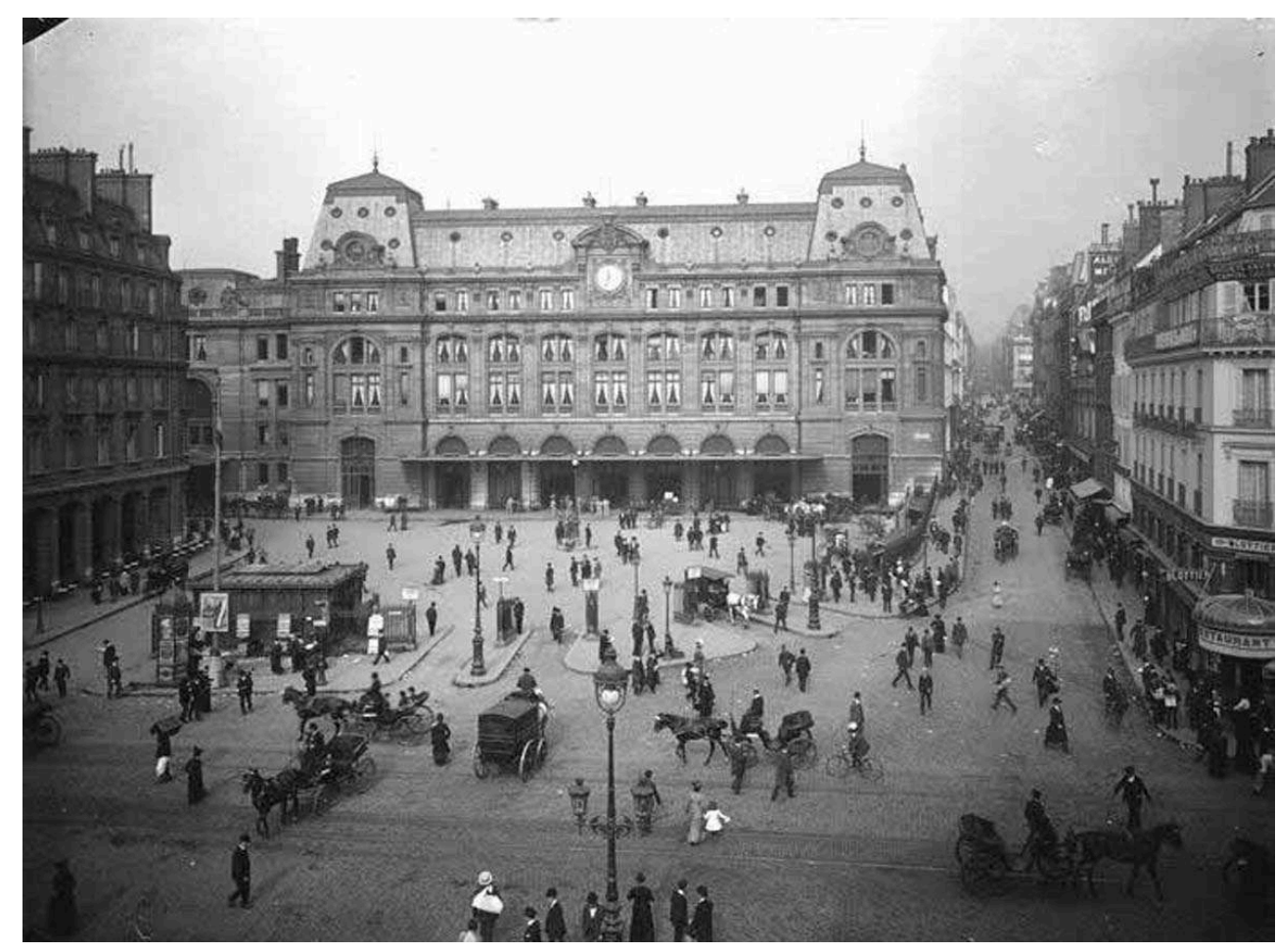

Paris, gare Saint-Lazare, cour du Havre

Phot. Séeberger (frères) s.d. [1er quart XXe s.]. Archives photographiques (Médiathèque de l'architecture et du patrimoine) (c) $\mathrm{CMN}$

La première ligne de chemin de fer française, reliant la gare Saint-Lazare à SaintGermain-en-Laye puis au Havre et à Dieppe a été inventoriée, à l'instar des travaux anglais de nos collègues d'English Heritage, par les chercheurs de l'Inventaire général et fera prochainement l'objet d'une publication conjointe des deux régions d'Ile-deFrance et de Haute-Normandie (fig. $\mathbf{n}^{\circ} \mathbf{2}$ ). Nous continuerons à explorer tout ce que l'extension du chemin de fer doit à la villégiature. Mais nous avons appris aussi, grâce à Paul Smith ${ }^{1}$, ce que lui doit le réseau routier, avec, par exemple, la première «stationservice » encore en place à Deauville. L'histoire de l'automobile, liée à celle de la grande bourgeoisie, est indissociable de l'urbanisme ainsi que de l'architecture (l'installation de garages et d'accès dans les villas) de la villégiature.

On voit ainsi se dessiner, sous le réseau «physique » des communications, un réseau social qu'on peut bien appeler un réseau "de classe», qui structure les stations, les relie entre elles et aux grandes villes ou à leurs banlieues résidentielles. Bernard Toulier cite parfois le cas de l'industriel Latécoère dont le nom est lié aux débuts de l'histoire de l'aviation française. A Toulouse, les hangars où il construisait des wagons de chemins de fer, réquisitionnés pendant la Grande Guerre sont aujourd'hui protégés ${ }^{2}$, ainsi que, dans la même ville, les vestiges de l'Aéropostale. Mais non loin des usines, la villa de Latécoère affichait ses somptueux jardins dont les allées se disposent comme les pistes d'un aérodrome ${ }^{3}$. Or, très loin de là, l'Inventaire général a retrouvé Latécoère dans sa villa du Pays basque, et l'on sait qu'il possédait aussi, en Touraine, le château du Coudray-Montpensier ${ }^{4}$. Voilà bien un cas où la recherche, si elle se limitait à une seule région, manquerait son objectif: seule l'échelle nationale, avec une confrontation comme celle que nous organisons aujourd'hui, peut rendre compte de ces réseaux 
sociaux - on pourrait dire ici "mondains » - qui associent l'architecture balnéaire, aéronautique, le patrimoine industriel et l'histoire récente des châteaux de la Renaissance. On pourrait citer aussi le chocolatier Menier avec ses usines de Noisiel, le village qui les entoure mais aussi le château de Chenonceau qui lui appartint et présente avec Noisiel de troublantes analogies (la construction sur une ancienne pile de moulin avec un pont qui enjambe la rivière) et l'île d'Anticosti au Canada qu'il avait transformée en terrain de chasse privé.

6 Le « réseau social » de la villégiature s'étend bien sûr aux architectes et aux artistes qui travaillaient pour cette clientèle "délocalisée ». On sait par les études de Claude Laroche $^{5}$ sur le Pays basque que, paradoxalement, on doit à cette délocalisation des propriétaires et de leurs architectes une bonne partie de ce qu'on appelle l'architecture "régionaliste », le néo-basque ou le néo-normand. François Fray ${ }^{6}$ a savamment étudié le réseau des clients que l'architecte américain Barry Dierks s'était constitué sur la Côte d'Azur après en avoir retrouvé les archives. Car ce réseau de producteurs d'architecture et d'urbanisme expérimentaux est vite devenu international. Gilles Ragot $^{7}$ nous a montré comment l'école brésilienne, courant majeur de l'architecture moderne, a pénétré en France à l'occasion de la reconstruction de Royan, d'une manière presque subreptice que seul, peut-être, un chantier de station balnéaire, avec ses bâtiments festifs ou somptuaires, permettait.

Figure 3

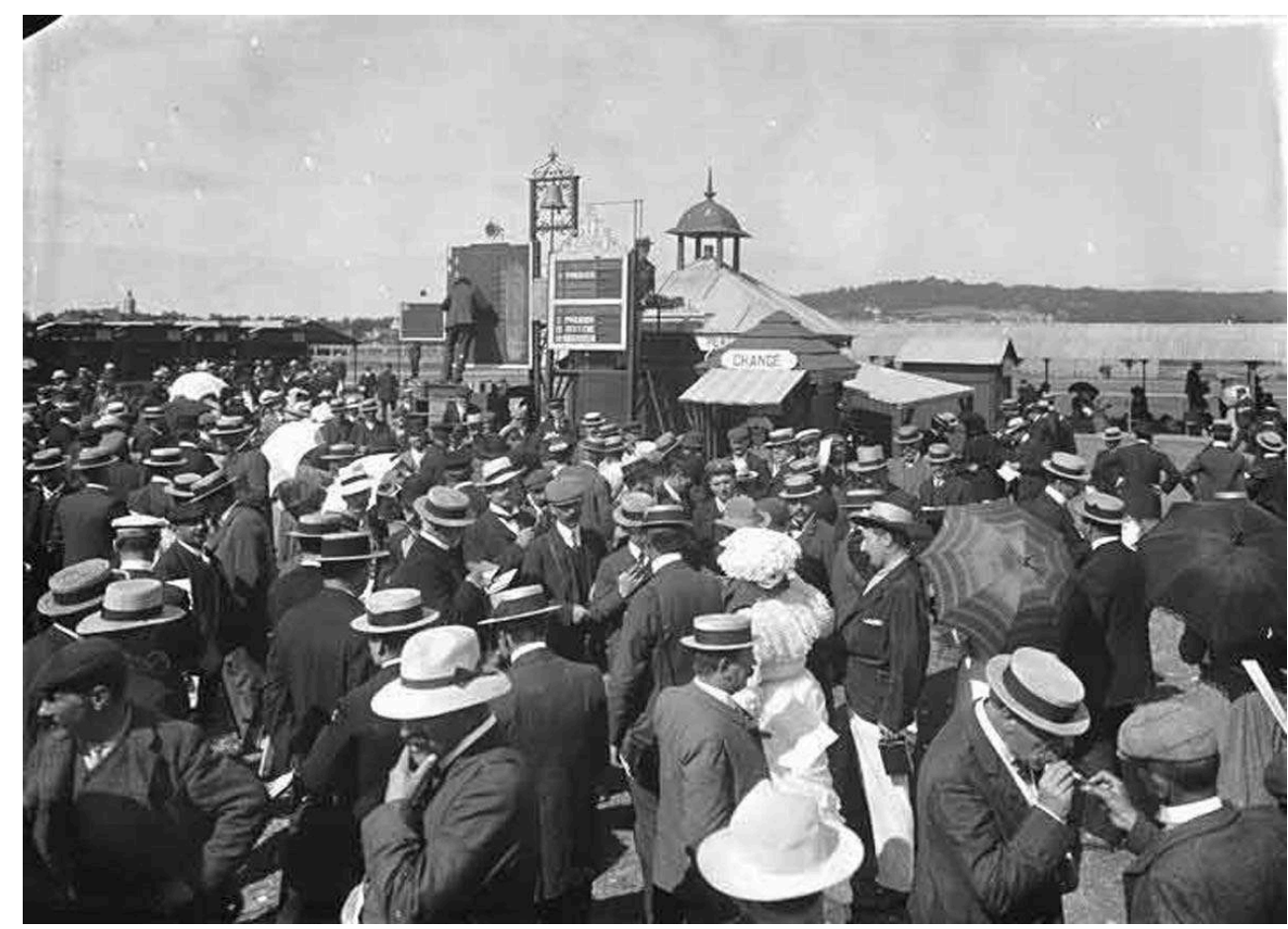

Courses à Deauville : L'affichage des résultats . Phot. Séeberger (frères) s.d. [1 ${ }^{\text {er }}$ quart XXe s.] Archives photographiques (Médiathèque de l'architecture et du patrimoine) (c) CMN

7 On doit aussi étendre ces relations aux arts décoratifs, dont l'architecture de villégiature est prodigue, et aux collections d'objets d'art qui peuplent les villas. J'avais été frappé, lors de nos rencontres de Deauville, tenues dans l'ancienne villa des Rothschild, aujourd'hui propriété de la ville, d'y voir une impressionnante collection de tableaux, tous sur le thème du cheval. Que l'art balnéaire ait un rapport aussi fort avec 
l'art hippique - comme elle en nourrit d'ailleurs avec beaucoup d'autres sports - mérite déjà réflexion mais on en verra toute l'importance pour l'urbanisme lorsqu'on sait que la petite commune de Deauville est pourvue non seulement d'un aéroport mais aussi de deux hippodromes (fig. $\mathbf{n}^{\circ} \mathbf{3}$ ).

Figure 4

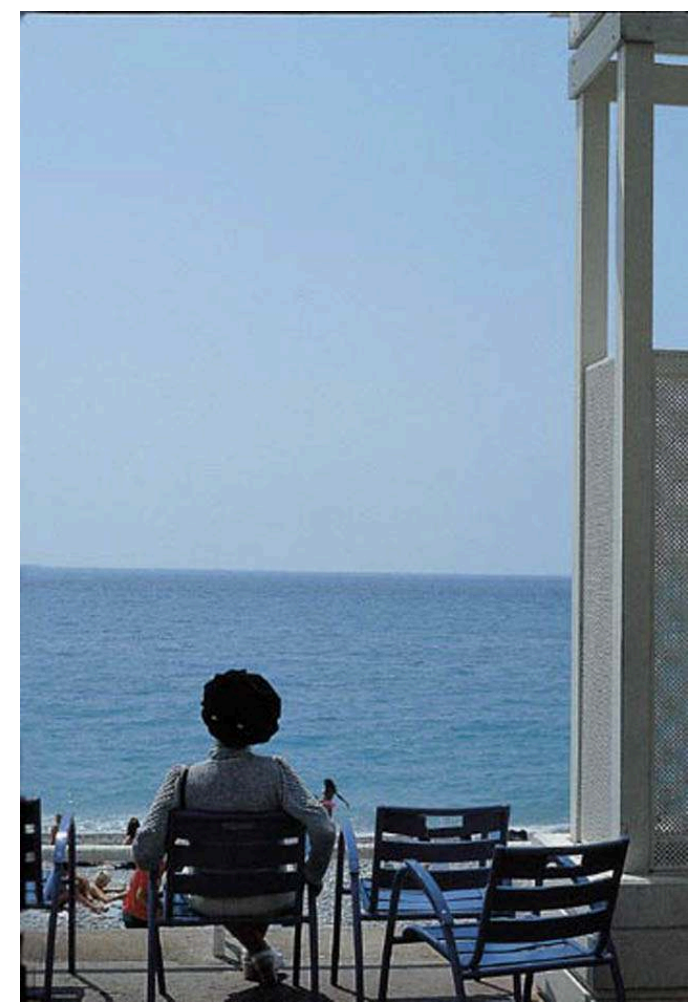

Nice, front de mer, Promenade des Anglais, face à la Méditerranée

Phot. C. Rose. Extrait : villes d'EAux, Paris : Imprimerie nationale Editions/Dexia, 2002, p. 8

Ces rapprochements sont si hétérogènes qu'on aurait tendance à négliger le cœur même de l'urbanisme balnéaire : la mer (fig. $\mathbf{n}^{\circ} \mathbf{4}$ ). La mer oriente l'habitat, détermine les axes urbains et les angles de vue, articule le parcellaire. Il est vrai qu'on dit que Deauville tourne le dos à la mer qui n'y est guère visible, mais ce n'est pas le cas de toutes les stations avec leurs promenades, leurs fronts de mer et leurs marinas. Il m'a semblé qu'on accordait trop peu de place au littoral dans ces études d'architecture dont l'urbanisme est malgré tout lié au port et au rivage qui ouvre ces cités vers l'outre-mer, rendant proches les ports les plus lointains et instituant un réseau naturel. Le réseau des stations balnéaires est d'ailleurs aussi bien celui qu'elle tisse tout aussi naturellement avec l'arrière-pays. La collision est violente entre les réseaux de transports à longue distance et ceux de proximité, car la villégiature, si lointaine soitelle, doit d'abord inviter à l'intimité et aux réseaux de voisinage et l'on y passe directement de l'autoroute au chemin de douanier, de l'avion au jardin. La démarche qui consisterait à n'étudier que l'architecture balnéaire du littoral est absurde: néanmoins, c'est ce que souhaitent parfois, avec de bonnes intentions, les communes qui souhaitent mettre en valeur leur patrimoine balnéaire. Or, le village ancien souvent en retrait, les communes de l'arrière-pays sont souvent le noyau de la villégiature et, aujourd'hui encore, son prolongement naturel, y compris sur la plan touristique. Il faut 
donc ici comme ailleurs ne pas extraire l'architecture de son contexte et respecter les méthodes de l'Inventaire général qui préconisent de privilégier l'approche topographique sur l'approche thématique.

Nous avons constaté ensemble une autre lacune dans nos études: celle des quartiers populaires de la villégiature. On trouve dans les stations balnéaires ou thermales, mais aussi dans les lieux populaires comme les villes de pèlerinage, un habitat social important destiné à loger le personnel des établissements, sachant que le personnel de service des villas était souvent logé, comme les serviteurs des cours princières, dans les galetas ou les soupentes. Qu'elle donne lieu ou non à des architecture spécifiques mineures, voire à un urbanisme particulier des quartiers périphériques de la station, cette partie ancillaire de l'architecture de villégiature ne doit pas être négligée comme elle l'est et doit être reliée à d'autres réseaux.

Figure 5

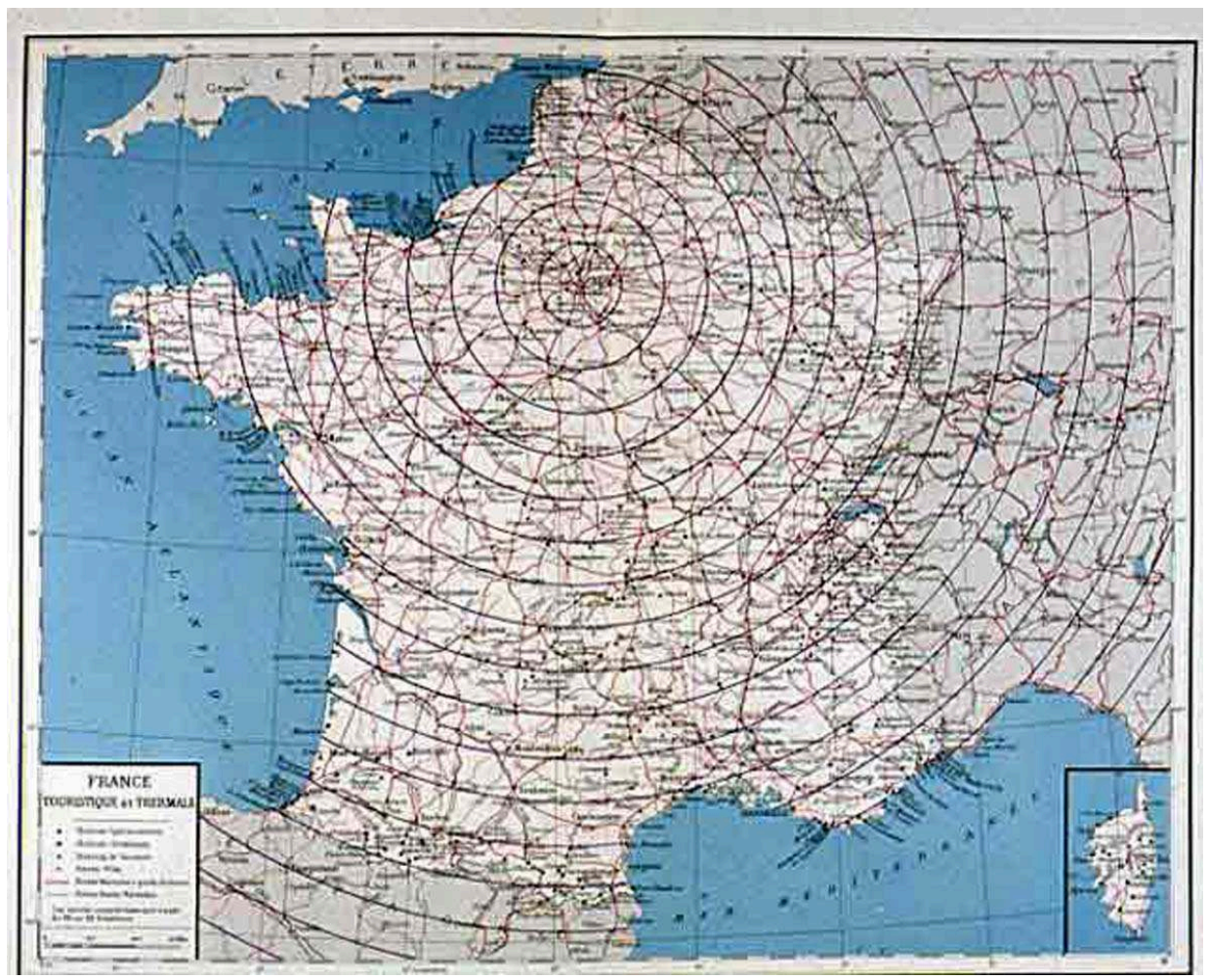

Carte de la France touristique et thermale. Del. Cdt. Pollachi, 1938

Coll. part. @ Droits réservés

A l'étude de ces réseaux superposés ou enchevêtrés doivent correspondre, de toute évidence, des réseaux de chercheurs qui en redessinent les traces parfois très éloignées les unes des autres par la distance mais aussi par le champ disciplinaire. Non seulement les historiens de l'architecture que nous sommes pour la plupart doivent mettre en commun leurs expériences - ce que nous faisons aujourd'hui - mais ils doivent les cumuler avec celles des géographes, des agronomes, des historiens de la société et de l'économie. Sans ces croisements, pas de typologie, pas de chronologie, pas d'explications sociales donc, pas d'histoire, au sens plein du terme, au-delà d'une érudition locale et complaisante. Aucune étude n'aurait de valeur sans les autres: Royan sans Brasilia, Deauville sans Honfleur, sans Paris ou sans Bruxelles, ou sans 
Atlantic City dont une étudiante française a entrepris d'écrire l'histoire et celle de sa "relance» qui témoigne de l'intérêt nouveau des Etats-Unis pour leur patrimoine balnéaire. Les réseaux de la villégiature nous invitent au voyage, dans l'espace social comme dans les territoires, bien au-delà de nos horizons (fig. $\mathbf{n}^{\circ}$ 5).

\section{NOTES}

1. Voir dans ce numéro l'article de Paul Smith : La place de l'automobile dans le développement des stations.

2. Usine aéronautique Latécoère de Montaudran à Toulouse : notice Mérimée PA31000012.

3. Parc dit domaine de Latécoère à Ramonville-Saint-Agne (31) : notice Mérimée IA31010081.

4. Domaine de Montpensier ou du Coudray-Montpensier à Seuilly (37): notice Mérimée PA00098114.

5. Voir dans ce numéro l'article de Claude Laroche : Hossegor (Landes), une station au cœur des réseaux de villégiature.

6. Voir dans ce numéro l'article de François Fray : La clientèle de l'architecte Barry Dierks sur la Côte d'Azur.

7. Voir dans ce numéro l'article de Gilles Ragot : L'invention du balnéaire « cinquante » à Royan.

\section{INDEX}

Mots-clés : réseaux, villégiature, gare Saint-Lazare, Saint-Germain-en-Laye, Le Havre, Dieppe, station-service, Latécoère, Aéropostale, Coudray-Montpensier (château), Noisiel, île d'Anticosti (Canada), Barry Dierks, Royan, Deauville, usine aéronautique Latécoère (Montaudran à Toulouse), Parc dit domaine de Latécoère (Ramonville-Saint-Agne), Domaine de Montpensier ou du Coudray-Montpensier (Seuilly)

\section{AUTEUR}

\section{MICHEL MELOT}

Ancien sous-directeur des études, de la documentation et de l'Inventaire de 1996 à 2003, Direction de l'architecture et du patrimoine. melotm@wanadoo.fr 